\title{
Qualitative Bilanzanalyse
}

Die qualitative Bilanzanalyse beschäftigt sich im Gegensatz zur quantitativen Bilanzanalyse mit der Auswertung der Bilanz und zugehöriger Komponenten in verbaler Form.

\subsection{Semantische und Syntaktische Analyse}

Die Analyse verbaler Informationen z. B. im Anhang (Erläuterungsbericht) oder Lagebericht kann unterschieden werden in semantische und syntaktische Analyse. Diese werden im Folgenden erläutert.

\section{Semantische Analyse}

Im Rahmen einer semantischen Analyse untersucht der Analyst die Anzahl der genutzten positiv besetzten Fachbegriffe (z. B. „Umsatzsteigerung“ und „Verbesserung") im Vergleich zu den negativ besetzten Fachtermini (z. B. „Verluste“, „Umsatzrückgang“). Die jeweils häufiger genutzten Fachbegriffe lassen entsprechend Rückschlüsse auf die tatsächliche Situation des Unternehmens ziehen.

\section{Syntaktische Analyse}

Gegenstand der syntaktischen Analyse ist die Betrachtung der ausgewählten Begriffe bzw. die Darstellung bestimmter Situationen.

So macht es durchaus einen Unterschied, ob in einer Bilanzanalyse von einer Gewinnminderung in Höhe von $10 \%$ gesprochen wird oder von einem leichten Gewinnrückgang. Während die prozentuale Angabe eine präzise Formulierung darstellt, kann man bei der zweiten Formulierung eine gegebenenfalls zu milde Darstellung unterstellen, die die tatsächliche wirtschaftliche Situation verzerrt darstellt. 